\title{
Lattice QCD with Chiral Chemical Potential: from SU(2) to SU(3)
}

\section{V. Braguta}

ITEP, Moscow, 117218 Russia

E-mail: braguta@itep.ru

\section{A. Goy}

FEFU, Vladivostok, 690950 Russia

E-mail: vovagoyegmail.com

\section{E-M. Ilgenfritz}

JINR, Dubna, Moscow region, 141980 Russia

E-mail: ilgenfri@theor.jinr.ru

\section{A. Yu. Kotov*}

ITEP, Moscow, 117218 Russia

E-mail: kotoveitep.ru

\section{A. V. Molochkov}

FEFU, Vladivostok, 690950 Russia

E-mail: molochkov.alexander@gmail.com

\section{Müller-Preussker}

Humboldt-Universität zu Berlin, Institut für Physik, Berlin, 12489 Germany

E-mail: mmp@physik. hu-berlin.de

\section{B. Petersson}

Humboldt-Universität zu Berlin, Institut für Physik, Berlin, 12489 Germany

E-mail: bengt@physik.hu-berlin.de

\section{S. A. Skinderev}

ITEP, Moscow, 117218 Russia

E-mail: sergeant.mipt@mail.ru

The phase diagram of two-color QCD with non-zero chiral chemical potential is studied by means of lattice simulation. We focus on the influence of a chiral chemical potential on the confinement/deconfinement phase transition and the breaking/restoration of chiral symmetry. The simulation is carried out with dynamical staggered fermions without rooting. The dependences of the Polyakov loop, the chiral condensate and the corresponding susceptibilities on the chiral chemical potential and the temperature are presented. We present here the first results of simulations with three-color QCD. The critical temperature is observed to increase with increasing chiral chemical potential for both gauge groups.

The 33rd International Symposium on Lattice Field Theory

14 - 18 July 2015

Kobe International Conference Center, Kobe, Japan* 


\section{Introduction}

For the vacuum state of QCD as well as for QCD at finite temperature the existence of nontrivial topological excitations is an important feature. Well known examples are instantons [1] and calorons $[2,3]$. The role of topologically charged fields for the solution of the famous $U_{A}(1)$ problem has been recognized very early $[4,5]$.

Some time ago the gluonic topological structure and the axial anomaly have been proposed to be immediately observable (and controllable) through the generation of $P$ and $C P$ violating domains in heavy ion collisions [6,7]. In this situation, the magnetic field created by the spectator nucleons may initiate a charge separation relative to the reaction plane (parallel to the magnetic field) [8]. The resulting charge asymmetry of quarks would become observable in terms of the recombined hadrons (chiral-magnetic effect) [9].

We report our study of the change of the phase structure induced by a chiral chemical potential $\mu_{5}$ in an equilibrium lattice simulation. The role of $\mu_{5}$ is to mimic the accumulated chiral imbalance due to a $C P$ violating, topologically nontrivial gluonic background in the early stadium of a heavy ion collision event, which is indirectly described by the chiral chemical potential. In this setting, the modification of the phase diagram by a chiral chemical potential $\mu_{5}$ has been studied mainly by means of effective models $[10,11,12,13,14,15,16]$. The predictions of these papers will later be compared with our results.

On the lattice, contrary to the case of non-zero baryon chemical potential, simulations with non-vanishing $\mu_{5}$ are not hampered by a sign problem. Thus, one can employ the standard hybrid Monte Carlo algorithms. Such lattice simulations with $\mu_{5} \neq 0$ were already performed in Ref. [17, 18]. The main goal of these papers, however, was the study of the chiral magnetic effect. Therefore, the phase diagram was not systematically studied.

In Refs. [19, 20, 21] we have carried out the first pilot lattice study of the phase diagram with non-zero chiral chemical potential. It was performed in $S U(2)$ QCD with four flavors, which we have considered as a simplified model of QCD.

One reason for choosing the $S U(2)$ gauge group was that less computational resources are required than for full QCD. The second reason is that we have already carried out two-colour QCD computations with an external magnetic field [22, 23] and plan to return to it with chemical potentials. Recently, we have started to run simulations with the $S U(3)$ gauge group and present here the first results of these measurements.

\section{Details of the simulations}

We have performed simulations with the $S U(2)$ gauge group. We employ the standard Wilson plaquette action. For the fermionic part of the action we use staggered fermions

$$
\begin{aligned}
S_{f}=m a & \sum_{x} \bar{\psi}_{x} \psi_{x}+\frac{1}{2} \sum_{x \mu} \eta_{\mu}(x)\left(\bar{\psi}_{x+\mu} U_{\mu}(x) \psi_{x}-\bar{\psi}_{x} U_{\mu}^{\dagger}(x) \psi_{x+\mu}\right)+ \\
& +\frac{1}{2} \mu_{5} a \sum_{x} s(x)\left(\bar{\psi}_{x+\delta} \bar{U}_{x+\delta, x} \psi_{x}-\bar{\psi}_{x} \bar{U}_{x+\delta, x}^{\dagger} \psi_{x+\delta}\right),
\end{aligned}
$$

* Speaker. 
where the $\eta_{\mu}(x)$ are the standard staggered phase factors: $\eta_{1}(x)=1, \eta_{\mu}(x)=(-1)^{x_{1}+\ldots+x_{\mu-1}}$ for $\mu=2,3,4$. The lattice spacing is denoted by $a$, the bare fermion mass by $m$, and $\mu_{5}$ is the value of the chiral chemical potential. In the chirality breaking term $s(x)=(-1)^{x_{2}}, \delta=(1,1,1,0)$ represents a shift to the diagonally opposite site in a spatial $2^{3}$ elementary cube. The combination of three links connecting sites $x$ and $x+\delta: \bar{U}_{x+\delta, x}=\frac{1}{6} \sum_{i, j, k=\operatorname{perm}(1,2,3)} U_{i}\left(x+e_{j}+e_{k}\right) U_{j}\left(x+e_{k}\right) U_{k}(x)$ is symmetrized over the 6 shortest paths between these sites.

In the continuum limit Eq. (2.1) can be rewritten in the Dirac spinor-flavor basis [24, 25] as follows, corresponding to a theory of four (degenerate) flavors

$$
S_{f} \rightarrow S_{f}^{(\text {cont })}=\int d^{4} x \sum_{i=1}^{4} \bar{q}_{i}\left(\partial_{\mu} \gamma_{\mu}+i g A_{\mu} \gamma_{\mu}+m+\mu_{5} \gamma_{5} \gamma_{4}\right) q_{i}
$$

We would like to emphasize that the chiral chemical potential, introduced in Eq. (2.1), corresponds to the taste-singlet operator $\gamma_{5} \gamma_{4} \otimes 1$ in the continuum limit.

We have performed simulations with two lattice sizes $N_{\tau} \times N_{\sigma}^{3}=6 \times 20^{3}, 10 \times 28^{3}$. The measured observables are: the Polyakov loop, the chiral condensate, the Polyakov loop susceptibility, the disconnected part of the chiral susceptibility. The Polyakov loop and the corresponding susceptibility are sensitive to the confinement/deconfinement phase transition, whereas the chiral condensate in principle responds to chiral symmetry breaking/restoration.

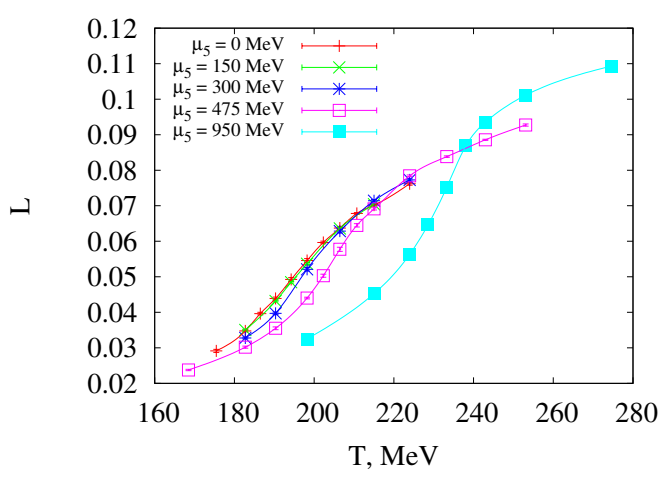

Polyakov loop

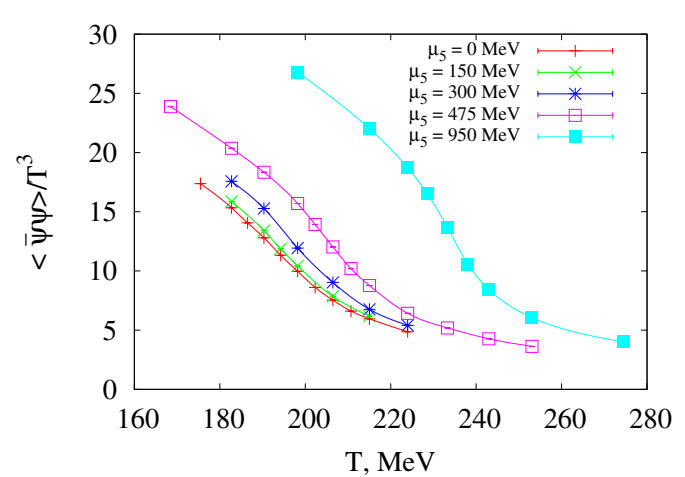

chiral condensate

Figure 1: Polyakov loop and chiral condensate versus $T$ for five values of $\mu_{5}$. Lattice size is $6 \times 20^{3}$, fermion mass is $m \approx 12 \mathrm{MeV}$. Errors are smaller than the data point symbols. The curves are to guide the eyes.

We use the results of Ref. [22] for the scale setting (calibration) of the lattice.

\section{Results of the calculation}

We present results of our simulations on a lattice of size $6 \times 20^{3}$ for five fixed values of $\mu_{5}=$ $0,150,300,475,950 \mathrm{MeV}$ and different values of $T$. The fermion mass was kept fixed in physical units $m \approx 12 \mathrm{MeV}\left(m_{\pi} \approx 330 \mathrm{MeV}\right)$. The expectation values of the Polyakov loop and the chiral condensate are shown in Fig. 1. The sharp change of the observables as functions of $T$ indicates the onset of the deconfinement and the chiral restoration phase transition. It is seen that the temperature 


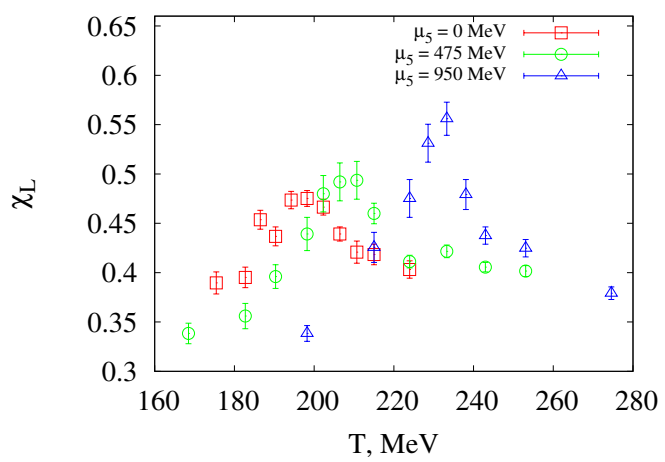

Polyakov loop susceptibility

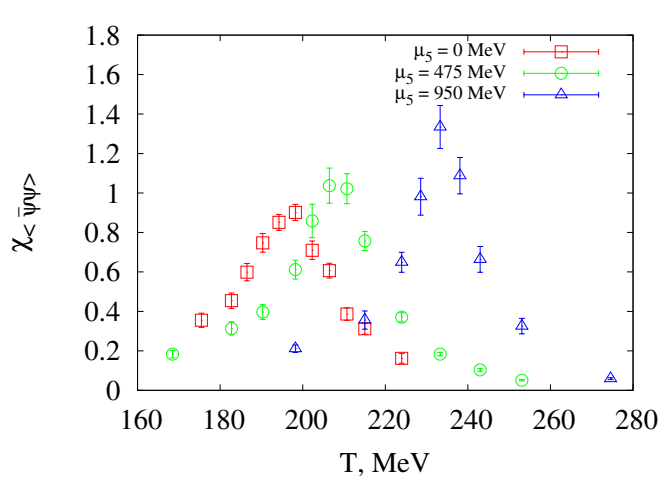

chiral susceptibility

Figure 2: Polyakov loop susceptibility and chiral susceptibility versus $T$ for three values of $\mu_{5}=0,475,950$ $\mathrm{MeV}$. Lattice size is $6 \times 20^{3}$, fermion mass is $m \approx 12 \mathrm{MeV}$. In order to avoid a complete superposition of data points belonging to different $\mu_{5}$ values we applied a tiny shift along the $T$ axis.

of both phase transitions increases with the chiral chemical potential. One also sees that the phase transition becomes sharper for increasing chiral chemical potential.

To study the change of the critical temperature more quantitatively, we also calculated the chiral and the Polyakov loop susceptibilities. The resulting dependences for values $\mu_{5}=0,475,950$ $\mathrm{MeV}$ are shown in Fig. 2 (in order to make the figure readable we do not present data for $\mu_{5}=$ $150,300 \mathrm{MeV}$ on it). We see that increasing the value of the chiral chemical potential moves the position of the peaks of the both susceptibilities to larger values of $\beta$. This means that the transition temperature increases. We have fitted the data for both the susceptibilities near the peak with a Gaussian function and extracted the critical temperatures $T_{c}^{\chi}\left(\beta_{c}^{\chi}\right)$ and $T_{c}^{L}\left(\beta_{c}^{L}\right)$. The resulting dependences of both critical temperatures on the value of the chiral chemical potential $\mu_{5}$ are shown on the Fig. 3. One sees that for all points except for $\mu_{5}=950 \mathrm{MeV}$ the critical temperatures $T_{c}^{\chi}$ and $T_{c}^{L}$ coincide within errors. There is a slight discrepancy for $\mu_{5}=950 \mathrm{MeV}$, but because of possible systematic uncertainties in the fit as well as finite size effects, we cannot claim that the transition

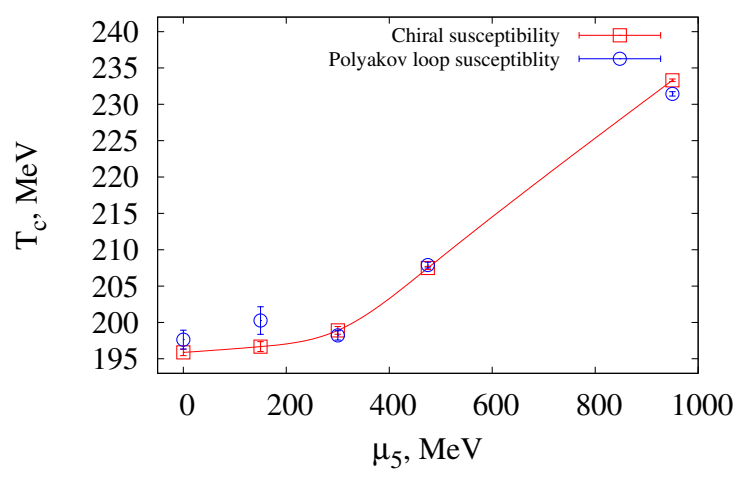

Figure 3: The dependence of the critical temperatures $T_{c}^{\chi}$ and $T_{c}^{L}$ on the value of the chiral chemical potential. Lattice size is $6 \times 20^{3}$, fermion mass is $m \approx 12 \mathrm{MeV}$. The curve connecting the points for $T_{c}^{\chi}$ is to guide the eyes. 
temperatures are different.

The dependence of the observables on the quark mass $m a$ was detailed studied in [21] on larger lattice $10 \times 28^{3}$. Extrapolation to zero quark mass allows us to conclude that the behaviour qualitatively is the same in the chiral limit: the critical temperature grows with $\mu_{5}$.

\section{First results for $S U(3)$ gauge group.}

We started to perform simulations with $S U(3)$ gauge group and dynamical Wilson fermions and present the first preliminary results of this study in Fig. 4 for five values of $\mu_{5} a=0,0.25,0.5,0.75$ and 1.00. The results show that in this case the critical $\beta$ determined by the sharp change in the observables increases with growing $\mu_{5}$, what means that the critical temperature also grows, thus being in agreement with our results for $S U(2)$ gauge group.

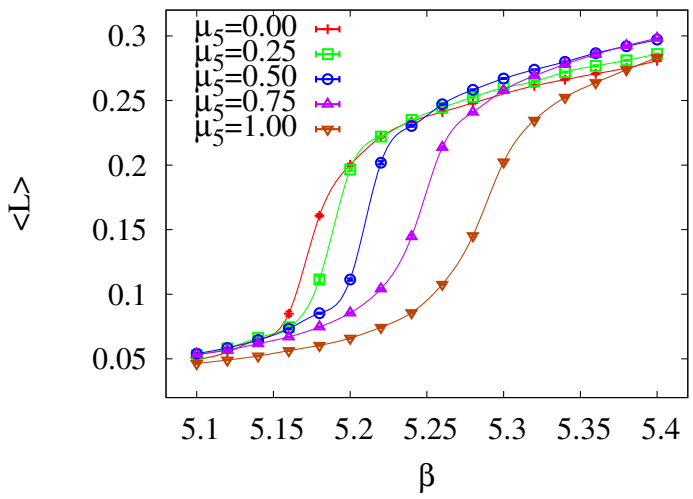

Polyakov loop

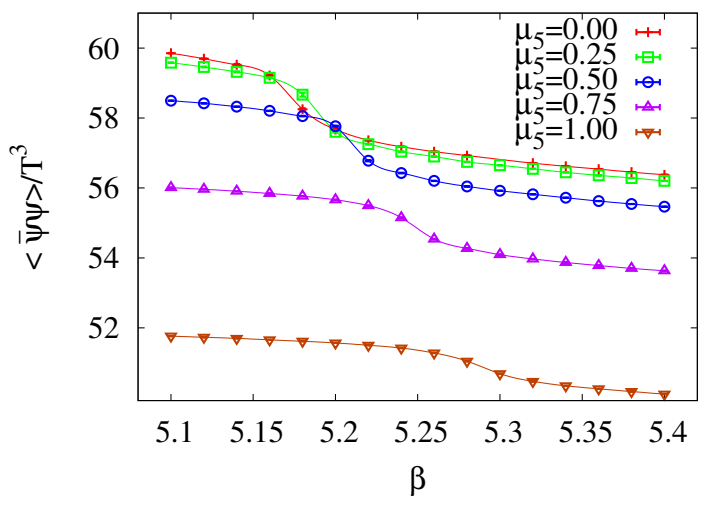

chiral condensate

Figure 4: Polyakov loop and chiral condensate versus $T$ for five values of $\mu_{5}$ for Wilson fermions and $S U$ (3) gauge group. Lattice size is $4 \times 16^{3}, \kappa=0.1665$. For $\beta=5.32144$ it corresponds to the pion mass $m_{\pi}=418 \mathrm{MeV}$. Errors are smaller than the data point symbols. The curves are to guide the eyes.

\section{Discussion and conclusion}

We have presented an investigation of the phase diagram of two-color QCD with a chiral chemical potential using lattice simulations with dynamical staggered fermions without rooting.

We have calculated the chiral condensate and the Polyakov loop for different values of the temperature $T$ and chiral chemical potential $\mu_{5}$ and their respective susceptibilities. Our main result is the observation that the finite temperature phase transition becomes clearly shifted to larger critical temperature with rising chiral chemical potential. It was seen that this conclusion remains true when one is extrapolating to vanishing quark mass. Additionally we saw the respective critical temperatures $T_{c}^{L}$ and $T_{c}^{\chi}$ to agree within errors at least up to $\mu_{5} \approx 0.5 \mathrm{GeV}$.

Our result is in contradiction to results obtained with various effective models of QCD [10, $11,12,15,16]$, where the critical temperature was said to decrease as $\mu_{5}$ increases.

We concede that we study two-color QCD with $N_{f}=4$ quarks which is different from what is mostly considered in the effective models. For a closer comparison we have started the lattice 
simulation for the $S U(3)$ gauge group and $N_{f}=2$ Wilson fermions and the first results are in agreement with the presented conclusions.

It is likely that the contradictions are rather a consequence of the fact that the critical temperature is not a universal parameter but crucially depends on the structure and parameters of the effective models. It is unclear to what extent they describe the actual behavior of finite temperature QCD. It should be also noted that some predictions obtained in different effective models are in conflict with each other.

In addition to the dependence of the critical temperature on the chiral chemical potential, some effective models predict that - beginning from some critical value of $\mu_{5}^{c}-$ the transition turns into a first order transition. In our simulations we see that the transition becomes sharper as we increase $\mu_{5}$, but we don't see a first order phase transition for our parameters.

Besides within effective models, the behaviour of QCD and QCD-like theories with nonzero $\mu_{5}$ was studied in papers [26, 27] in a framework of Dyson-Schwinger equations and in [28] from the point of view of large- $N_{c}$ equivalence. The authors of these papers found that the critical temperature rises with $\mu_{5}$ and the "phase transition" actually is always a crossover. These results are corroborating the results of our paper.

\section{Acknowledgements}

The authors are grateful to V. I. Zakharov and V. G. Bornyakov for interesting and stimulating discussions. The authors are grateful to N. Yamamoto who directed our attention to universality in large- $N_{c}$ QCD-like theories. The simulations were performed at GPUs of the K100 supercomputer of the Institute of Applied Mathematics of the Russian Academy of Sciences in Moscow and at GPUs of the Particle Phenomenology Group at the Institute of Physics of the Humboldt University Berlin. The work was supported by Far Eastern Federal University, by RFBR grants 13-02-01387a, 14-02-01185-a, 15-02-07596-a and 15-32-21117, by a grant of the president of the RF, MD3215.2014.2, and by a grant of the FAIR-Russia Research Center. The work of A. Yu. Kotov was also partially supported by the Dynasty foundation.

\section{References}

[1] A. A. Belavin, et al., Pseudoparticle solutions of the Yang-Mills equations, Phys. Lett. B59 (1975) 85.

[2] T. C. Kraan and P. van Baal, Periodic instantons with nontrivial holonomy, Nucl.Phys. B533 (1998) 627-659, [hep-th/9805168].

[3] K.-M. Lee and C.-H. Lu, SU(2) calorons and magnetic monopoles, Phys.Rev. D58 (1998) 025011, [hep-th/9802108].

[4] E. Witten, Current algebra theorems for the U(1) 'goldstone boson', Nucl. Phys. B156 (1979) 269.

[5] G. Veneziano, U(1) without instantons, Nucl. Phys. B159 (1979) 213.

[6] D. E. Kharzeev, Parity violation in hot QCD: Why it can happen, and how to look for it, Phys.Lett. B633 (2006) 260, [hep-ph / 0406125$].$

[7] K. Fukushima, D. E. Kharzeev, and H. J. Warringa, The Chiral Magnetic Effect, Phys.Rev. D78 (2008) 074033, [arXiv:0808.3382]. 
[8] D. E. Kharzeev, L. D. McLerran, and H. J. Warringa, The Effects of topological charge change in heavy ion collisions: 'Event by event P and CP violation', Nucl.Phys. $\mathbf{A 8 0 3}$ (2008) 227, [arXiv:0711.0950].

[9] STAR Collaboration Collaboration, B. I. Abelev et al., Azimuthal charged-particle correlations and possible local strong parity violation, Phys.Rev.Lett. 103 (2009) 251601, [arXiv: 0909. 1739].

[10] K. Fukushima, M. Ruggieri, and R. Gatto, Chiral magnetic effect in the PNJL model, Phys.Rev. D81 (2010) 114031, [arXiv:1003.0047].

[11] M. N. Chernodub and A. S. Nedelin, Phase diagram of chirally imbalanced QCD matter, Phys.Rev. D83 (2011) 105008, [arXiv: 1102 .0188].

[12] R. Gatto and M. Ruggieri, Hot quark matter with an axial chemical potential, Phys.Rev. D85 (2012) 054013, [arXiv:1110.4904].

[13] A. A. Andrianov, D. Espriu, and X. Planells, Chemical potentials and parity breaking: the Nambu-Jona-Lasinio model, Eur.Phys.J. C74 (2014) 2776, [arXiv: 1310 . 4416 ].

[14] X. Planells, et al., An effective theory for QCD with an axial chemical potential, PoS QFTHEP2013 (2013) 049, [arXiv:1310.4434].

[15] J. Chao, P. Chu, and M. Huang, Inverse magnetic catalysis induced by sphalerons, Phys.Rev. D88 (2013) 054009, [arXiv:1305.1100].

[16] L. Yu, H. Liu, and M. Huang, Spontaneous generation of local CP violation and inverse magnetic catalysis, Phys.Rev. D90 (2014), no. 7 074009, [arXiv: 1404.6969$].$

[17] A. Yamamoto, Chiral magnetic effect in lattice QCD with a chiral chemical potential, Phys.Rev.Lett. 107 (2011) 031601, [arXiv:1105.0385].

[18] A. Yamamoto, Lattice study of the chiral magnetic effect in a chirally imbalanced matter, Phys.Rev. D84 (2011) 114504, [arXiv:1111.4681].

[19] V. Braguta, et al., Study of the phase diagram of SU(2) quantum chromodynamics with nonzero chirality, JETP Lett. 100 (2015), no. 9 547-549.

[20] V. Braguta, et al., Two-Color QCD with Chiral Chemical Potential, PoS LATTICE 2014, 235 (2015) [arXiv: 1411.5174$]$.

[21] V. V. Braguta, et al., Two-Color QCD with Non-zero Chiral Chemical Potential, JHEP 1506, 094 (2015) [arXiv:1503.06670].

[22] E.-M. Ilgenfritz, et al., Two-color QCD with staggered fermions at finite temperature under the influence of a magnetic field, Phys.Rev. D85 (2012) 114504, [arXiv: 1203.3360].

[23] E.-M. Ilgenfritz, et al., Magnetic catalysis (and inverse catalysis) at finite temperature in two-color lattice QCD, Phys.Rev. D89 (2014) 054512, [arXiv: 1310 . 7876].

[24] H. Kluberg-Stern, et al., Flavors of Lagrangian Susskind Fermions, Nucl.Phys. B220 (1983) 447.

[25] I. Montvay and G. Münster, Quantum fields on a lattice. Cambridge University Press, 1994.

[26] B. Wang, et al., Effect of the chiral chemical potential on the position of the critical endpoint, Phys.Rev. D91 (2015), no. 3034017.

[27] S.-S. Xu, et al., Chiral phase transition with a chiral chemical potential in the framework of Dyson-Schwinger equations, Phys.Rev. D91 (2015), no. 5056003.

[28] M. Hanada and N. Yamamoto, Universality of phase diagrams in QCD and QCD-like theories, PoS LATTICE2011 (2011) 221, [arXiv: 1111 . 3391]. 